\title{
Dental Segmentation in Cone-beam Computed Tomography Images Using Watershed and Morphology Operators
}

\begin{abstract}
Teeth segmentation is an important task in computer-aided procedures and clinical diagnosis. In this paper, we propose an accurate and robust algorithm based on watershed and morphology operators for teeth and pulp segmentation and a new approach for enamel segmentation in cone-beam computed tomography (CBCT) images. Proposed method consists of five steps: acquiring appropriate CBCT image, image enhancement, teeth segmentation using the marker-controlled watershed (MCW), enamel segmentation by global threshold, and finally, utilizing the MCW for pulp segmentation. Proposed algorithms evaluated on a dataset consisting 69 patient images. Experimental results show a high accuracy and specificity for teeth, enamel, and pulp segmentation. MCW algorithm and local threshold are accurate and robust approaches to segment tooth, enamel, and pulp tissues. Methods overcome the over-segmentation phenomenon and artifacts reduction.
\end{abstract}

Keywords: Dental cone-beam computed tomography, marker-controlled watershed, morphology operators, segmentation

\section{Introduction}

Cone-beam computed tomography (CBCT) imaging is an effective volumetric diagnosis imaging technology that produces accurate, submillimeter-resolution images of the maxillofacial region for dental practitioners to perform multiplaner imaging and provide three-dimensional (3D) information. ${ }^{[1]}$ In modern dentistry, computer-aided procedures such as surgical preoperative planning, mechanized dental implant, orthodontic planning, and other common procedures are getting more attentions day by day. ${ }^{[2]}$ Dental segmentation is one of the most important steps in computer-aided procedures. It can produce approximate outlines of doubtful regions to provide features which enable distinction between tooth tissues and other tissues. Since teeth boundaries are usually obscured, irregular, and low contrast, teeth segmentation is a challenging task. In recent years, a number of methods have been developed for teeth segmentation. Hosntalab et al. presented a segmentation approach for CT volumetric dataset. They calculated the integral projection and used Butterworth filter for noise reduction. This technique was effective to acquire an accurate and smooth teeth

This is an open access journal, and articles are distributed under the terms of the Creative Commons Attribution-NonCommercialShareAlike 4.0 License, which allows others to remix, tweak, and build upon the work non-commercially, as long as appropriate credit is given and the new creations are licensed under the identical terms.

For reprints contact: reprints@medknow.com contour. ${ }^{[3]}$ Momeni and Aghaeizadeh Zoroofi introduced an automated method based on wavelet descriptors to dental recognition in multislice computed tomography (MSCT) data. Experimental results reveal that their proposed technique is effective to automatically teeth classification. ${ }^{[4]}$ Akhoondali et al. used a 3D median filter to reduce noise in CT data. Then, weight-masked image from threshold filter was applied for metal artifact reduction. Finally, they used maximum intensity projection and region growing for teeth segmentation. Results showed the high accuracy and verified to be a good tool for identification of human. ${ }^{[5]}$ In Sepehrian et al. study, ${ }^{[6]}$ a watershed segmentation algorithm in CBCT and MSCT images was presented. Results show a high accuracy for proposed algorithm in segmenting teeth. Mortaheb et al. proposed a fuzzy $\mathrm{C}$-mean clustering based thresholding and least square support vector machine for dental segmentation in CBCT images. Mean shift filter applied to remove noise. Result approach shows a high specificity and accuracy to proposed technique. ${ }^{[7]}$ In this study, we propose accurate hybrid methods based on watershed and morphology operators and local threshold method for teeth, enamel, and pulp segmentation in

\footnotetext{
How to cite this article: Kakehbaraei $S$, Seyedarabi H, Zenouz AT. Dental segmentation in cone-beam computed tomography images using watershed and morphology operators. J Med Sign Sens 2018;8:119-24.
}

\section{Somayeh \\ Kakehbaraei ${ }^{1}$, Hadi Seyedarabi ${ }^{1,2}$, Ali Taghavi Zenouz ${ }^{3}$}

${ }^{1}$ Department of Biomedical Engineering, Faculty of Advanced Medical Sciences, Tabriz University of Medical Sciences, Tabriz, Iran, ${ }^{2}$ Faculty of Electrical and Computer Engineering, University of Tabriz, Tabriz, Iran, ${ }^{3}$ Department of Oral Medicine, Faculty of Dentistry, Tabriz University of Medical Sciences, Tabriz, Iran 
CBCT images. The focus is mainly on noise reduction, overcomes the over-segmentation, and gets more accuracy for detecting teeth and pulp contours and enamel regions, which are important tasks in the segmentation methods; also, dental caries and dental damage can be detected using enamel and pulp segmentation.

\section{Methods}

\section{Dataset}

CBCT scans were performed on a NewTom system. The dataset contains 69 patient images that from this dataset, for each of the segmentation algorithms, 30 images have been selected. The images are stored in DICOM format with dimensions of $512 \times 512$ pixels and taken in axial position.

\section{Mathematical morphology}

Morphology is a domain of image processing which is mainly useful for analyzing region shapes in images such as boundaries and skeletons. ${ }^{[8]}$ There are several morphological tools in image segmentation consisting erosion, dilation, reconstruction, etc.

\section{Dilation and erosion}

The functions of dilation and erosion are primary to morphological image processing. ${ }^{[8]}$ Mathematically, dilation is defined in terms of set operations. The dilation of $I$ by $\mathrm{G}$ denoted as $I \oplus \mathrm{G}$, is defined as:

$I \oplus G=\{z \mid(\hat{G}) \mathrm{z} \cap I \neq \varnothing\}$

Where $\varnothing$ is empty set and $G$ is the structuring element. The erosion $I$ by $G$, denoted $I \Theta G$, is defined as:

$I \Theta G=\left\{z \mid(G) \mathrm{z} \cap I^{\mathrm{c}} \neq \varnothing\right\}$

Where $I^{\mathrm{c}}$ is the complement of $I .^{[8]}$

\section{H-maxima transform}

H-maxima transform suppresses all regional maxima in the gradient image. Regional maxima are connected components of pixels with a constant intensity value, and whose external boundary pixels all have a lower value. ${ }^{[9]}$

\section{Impose minima}

Impose minima transform modifies a gray-scale image so that regional minima appear only in marked locations. Other pixel values a "pushed up" as essential to eliminate all other regional minima. ${ }^{[10]}$

\section{Marker-controlled watershed}

Marker-controlled watershed (MCW) used to control over-segmentation based on the concept of markers and segmentation of objects with closed contours, where the boundaries are expressed as ridges. The marker image used for watershed segmentation is a connected component belonging to an image. ${ }^{[8]}$

\section{Proposed work}

In this section, we propose details of the following five stages: (1) selecting appropriate CBCT image, (2) image enhancement, (3) teeth segmentation using the MCW, (4) enamel segmentation by global threshold, and (5) utilizing the MCW for pulp segmentation. Details of each step will be explained in the following sections. The block diagram of the suggested techniques is displayed as Figure 1.

Selecting appropriate cone-beam computed tomography image

We first determine the slice which includes teeth tissues and bony structures to reduce complexity and decrease processing time. In this case, an image that contains most teeth tissues is selected by a radiology specialist. This slice is saved in DICOM format.

\section{Image enhancement}

In CBCT images, there exists a certain amount of quantum noise, Moire artifacts, beam hardening, scatter, and cupping artifacts. ${ }^{[1]}$ Direct application of the watershed to an image usually leads to over-segmentation due to noise and other local irregularities of the image. ${ }^{[8]}$ Hence, without any enhancement, watershed algorithm cannot separate teeth, enamel, and pulp correctly. A 5-by-5 median filter is used to reduce artifacts and smooth the image before performing the segmentation

\section{Teeth segmentation}

Morphological operators were applied in three steps. First step is image filling to eliminate holes in teeth and unnecessary split line which can cause to wrong segmentation. In the second and third steps, morphology processing (dilation and erosion) was applied to obtain uniform intensity on teeth. The structure element used in this method is square shaped. Then, we utilize the Canny edge detector with a specified standard deviation in each image to obtain smooth image and detect edges.

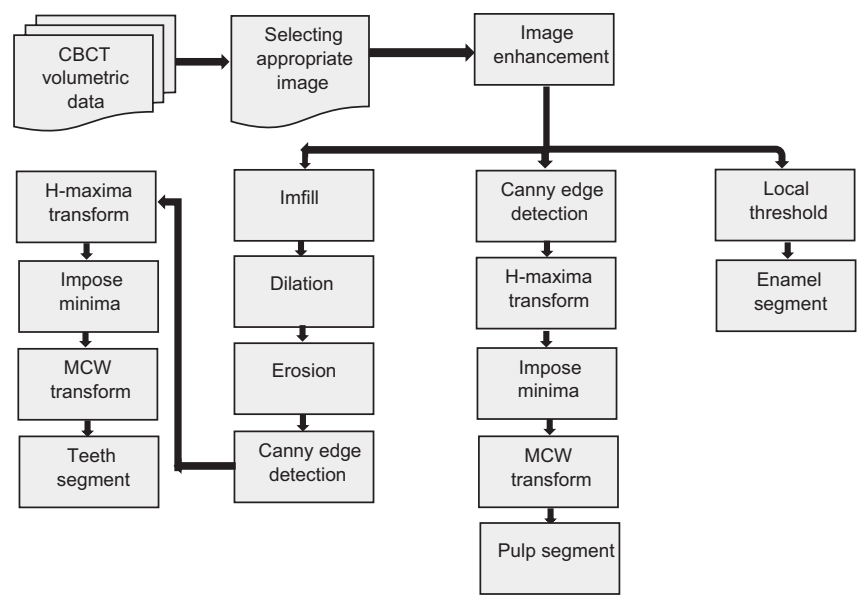

Figure 1: The block diagram of the suggested techniques 
Many regional minimas and maximas may exist in the morphological gradient image. These regionals may lead to over-segmentation in the watershed method. To clean up the gradient image, specified global threshold, H-maxima transform, and impose minima are employed in this study (Figure 2).

Finally, we complete segmentation process by applying the MCW algorithm on the marker-modified gradient image. Figure 3 shows this procedure.

In this method, marker image is defined as

$$
\text { Marker image }=A \oplus b
$$

Where $A$ is the boundary pixels of the objects in a morphological gradient image and $b$ is the 3-by-3 matrix of ones, which objects denote the boundary objects identified in the gradient image, such as the teeth and pulp.

\section{Enamel segmentation}

The enamel cap characteristically appears more radiopaque than the other tissues because it is the densest, naturally occurring substance in the body. ${ }^{[1]}$ For enamel segmentation, after obtaining appropriate image, we apply a 3-by-3 median filter to reduce noise. Finally, we utilize the local threshold for enamel segmentation. The procedure for the accurate enamel diagnosis is displayed in Figure 4.

\section{Pulp segmentation}

In this section, we introduce a new algorithm based on $\mathrm{MCW}$ for pulp segmentation. After acquiring appropriate image, we utilize a 3-by-3 median filter for noise reduction in CBCT images and the Canny edge detector to obtain a smoother image and the edge of objects in the image is applied. To remove the regional minimas and maximas in gradient image which leads to the phenomenon of over-segmentation, constant threshold, H-maxima transform, and impose minima are applied. The marker image used in this method is the marker image defined in the teeth segmentation step. Finally, we compute the watershed transform of the marker-modified gradient image. Figures 5 and 6 show the accurate diagnosis of pulp.

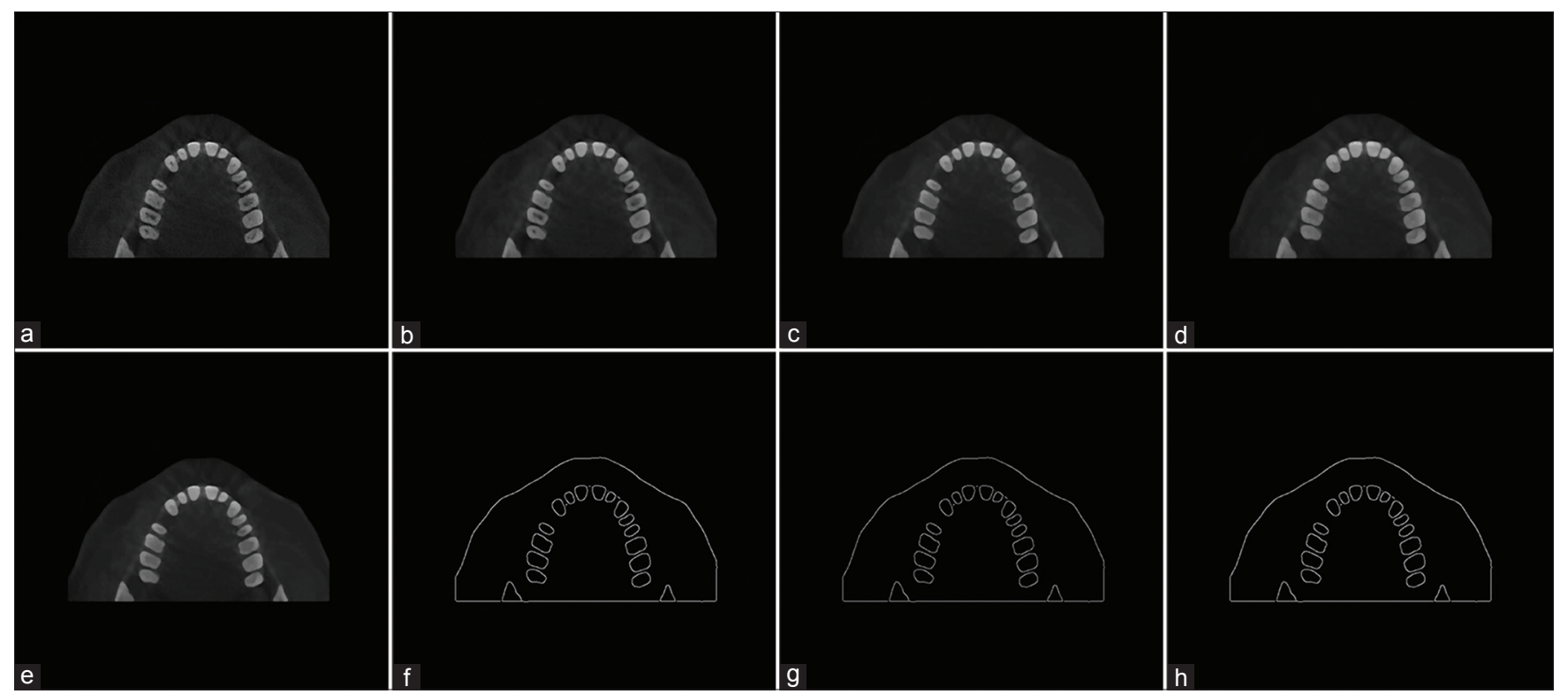

Figure 2: Preprocessing for teeth segmentation. (a) Selecting appropriate image. (b) Image filtering. (c) Filling holes in image. (d and e) Morphological operating for obtaining uniform intensity in image. (f) Applying Canny edge detector. (g and h) H-maxima transform and impose minima to remove regional minimas and maximas
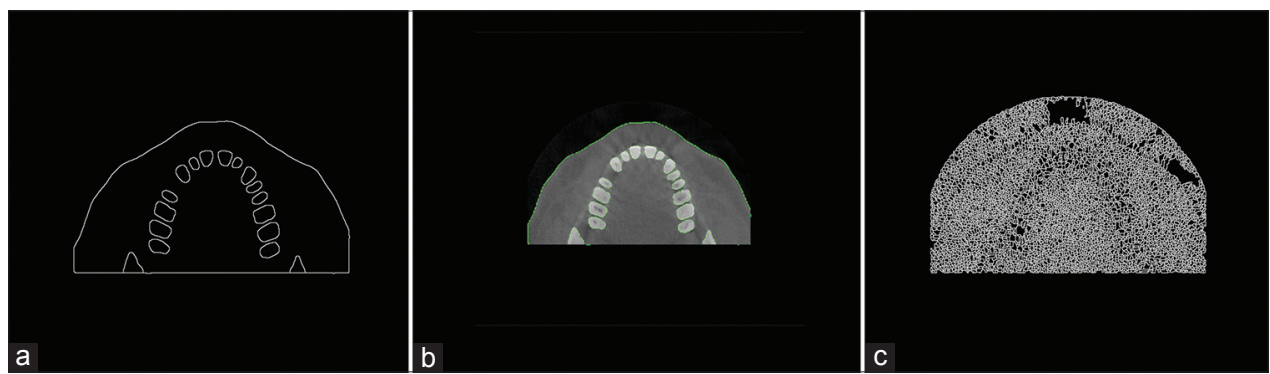

Figure 3: Segmentation results using proposed marker-controlled watershed algorithm. (a) Teeth segmentation. (b) Accommodating teeth boundary and marker-controlled watershed method. (c) Segmentation result without preprocessing 


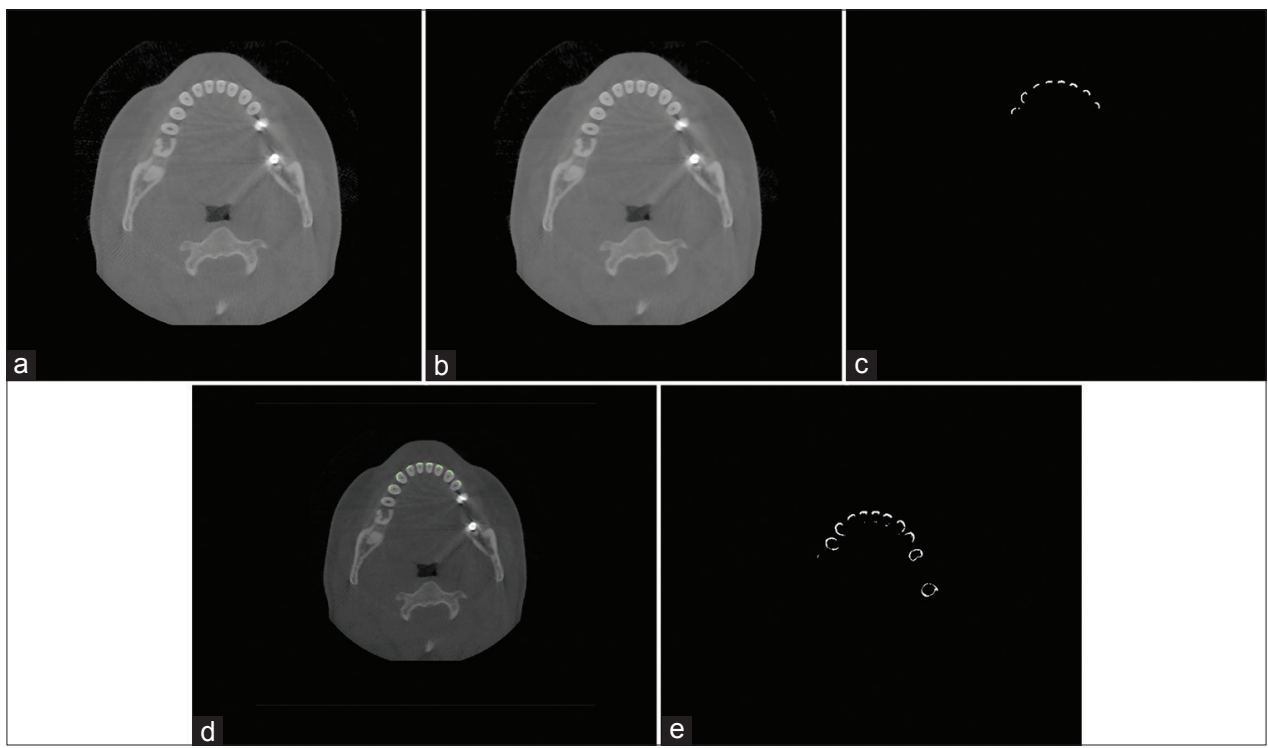

Figure 4: The process of the correct enamel diagnosis. (a) Selecting appropriate image. (b) Image filtering. (c) Accurate diagnosis of enamel. (d) Accommodating enamel areas and enamel segmentation method. (e) Segmentation result without filtering

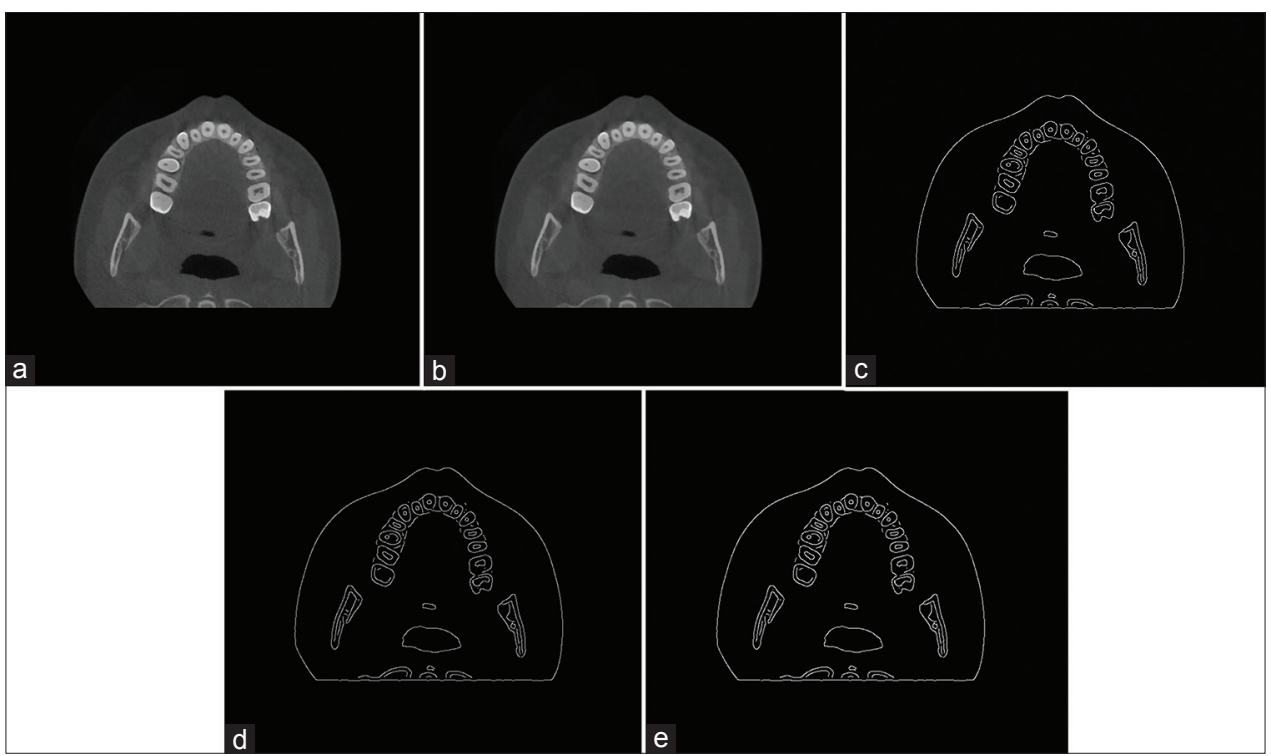

Figure 5: Preprocessing to correct pulp diagnosis. (a) Selecting appropriate image. (b) Image filtering. (c) Applying Canny edge detector. (d and e) H-maxima transform and impose minima to remove regional minimas and maximas

\section{Results}

The algorithms were implemented in MATLAB R2012b software package. ${ }^{[9]}$ The dataset contains various dental structures and forms. To evaluate the proposed methods, dataset was segmented by an expert manually, and these were considered as gold standard. In this regard, four types of pixels were computed as follows:

- True-positive pixels $\left(P_{\mathrm{TP}}\right)$ : Correctly segmented as dental tissues (teeth, enamel, and pulp)

- False-positive pixels $\left(P_{\mathrm{FP}}\right)$ : Incorrectly segmented as dental tissues (teeth, enamel, and pulp)

- False-negative pixels $\left(P_{\mathrm{FN}}\right)$ : Incorrectly segmented as nondental tissues
- True-negative pixels $\left(P_{\mathrm{TN}}\right)$ : Correctly segmented as nondental tissues

- $P_{\mathrm{REF}}$ : sum of $P_{\mathrm{TP}}, P_{\mathrm{TN}}, P_{\mathrm{FP}}$, and $P_{\mathrm{FN}}$.

The evaluation metrics are used in each image as follows: ${ }^{[1]}$

$$
\begin{aligned}
& \text { Sensitivity }=\left(\frac{P_{\mathrm{TP}}}{P_{\mathrm{TP}}+P_{\mathrm{FN}}} \times 100\right) \\
& \text { Specificity }=\left(\frac{P_{\mathrm{TN}}}{P_{\mathrm{TN}}+P_{\mathrm{FP}}} \times 100\right) \\
& \text { Accuracy }=\left(\frac{P_{\mathrm{TN}}+P_{\mathrm{TP}}}{P_{\mathrm{REF}}} \times 100\right) \\
& \text { Precision }=\left(\frac{P_{\mathrm{TP}}}{P_{\mathrm{TP}}+P_{\mathrm{FP}}} \times 100\right)
\end{aligned}
$$



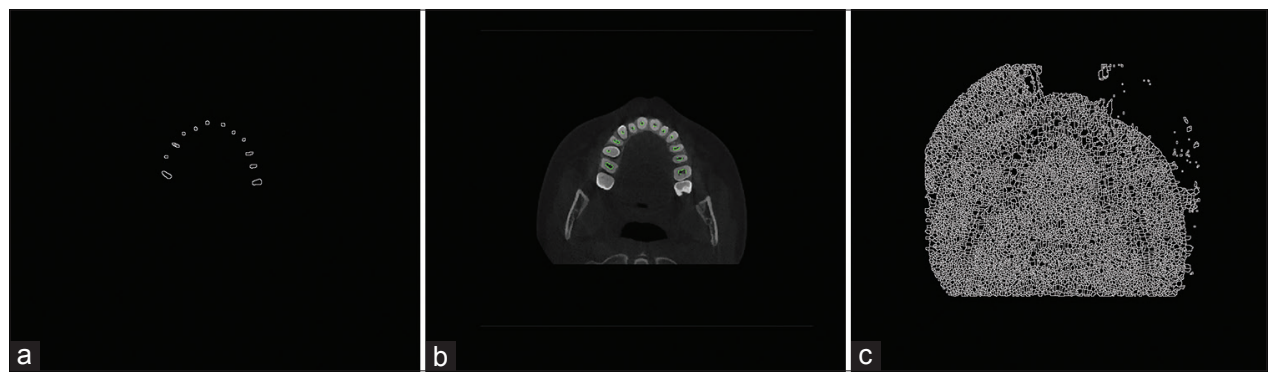

Figure 6: (a) Accurate diagnosis of pulp. (b) Accommodating pulp boundary and marker-controlled watershed method. (c) Segmentation result without preprocessing

And

False-positive rate $=\left(\frac{P_{\mathrm{FP}}}{P_{\mathrm{FP}}+P_{\mathrm{TN}}} \times 100\right)$

Evaluation results between the proposed methods and the gold standard are shown in Table 1.

\section{Discussion}

In this study, we introduce a new algorithm in teeth segmentation which is comparable in sensitivity, specificity, and accuracy with the previous works. ${ }^{[5,7]}$ As shown in Table 2, the rate of sensitivity, specificity, and accuracy of the proposed method is higher than other methods.

Our method increased the sensitivity, specificity, and accuracy of the segmentation results by $94.14 \%, 99.94 \%$, and $99.93 \%$, respectively, and also the number of images used in our paper is greater, which increases the accuracy and specificity of our method. In this paper, we overcome the over-segmentation phenomenon. In the cases with overlaying teeth, the adjacency of incisor, molar, and premolar teeth, CBCT image artifacts, and low-quality images, teeth segmentation algorithm failed to produce precision results. In several images, bones were considered as part of the tooth because of imprecise segmentation. We experientially found that the segmentation of teeth in the mandible is more difficult than maxilla because the mandibular shape is complex. In addition, CBCT image artifacts and low-quality images are the major obstacles in dental segmentation. To analyze the results of enamel and pulp segmentation, we calculated the correct diagnosis rate of enamel and pulp. In some images with dental implant and metallic structures such as amalgam fillings, these regions were considered as part of the enamel because of light scattering caused by metal structures. In this stage, the operator may delete surplus segmentation lines and regions. We were not able to compare the results of enamel and pulp segmentation with other studies because the related work has not been done in this context.

\section{Conclusion}

In this study, we propose an accurate and robust MCW algorithm to segment tooth and pulp tissues and an accurate method to segment enamel in CBCT images. We overcome the phenomenon of over-segmentation and artifact reduction.

\begin{tabular}{lccccc}
\hline \multicolumn{6}{c}{ Table 1: } \\
\hline Target & Sensitivity & Specificity & Accuracy & Precision & FPR \\
\hline Teeth & 94.14 & 99.94 & 99.93 & 86.81 & 0.06 \\
Enamel & 80.35 & 99.98 & 99.94 & 91.32 & 0.02 \\
Pulp & 83.30 & 99.99 & 99.98 & 91.00 & 0.01 \\
\hline FPR - False-positive rate
\end{tabular}

Table 2: The comparison of our method with other methods

\begin{tabular}{lcccc}
\hline Methods & Sensitivity & Specificity & Accuracy & Precision \\
\hline Akhoondali et.al. ${ }^{[5]}$ & 90 & 98 & 97 & 94 \\
Mortaheb and $_{\text {Rezaeian }}^{[7]}$ & 83.24 & 98.35 & 97.62 & 72.77 \\
Proposed method & 94.14 & 99.94 & 99.93 & 86.81 \\
\hline
\end{tabular}

Proposed methods provide more accurate and robust segmentation results for teeth, enamel, and pulp regions from other regions. Teeth segmentation can be used to improve the location information efficiency for orthodontic treatment, implant planning, and problems of decision support in medical diagnosis such as human identification and maxillofacial surgery. The future plans include finding new methods to artifacts reduction, diagnosis dental caries, and an automatic segmentation technique to provide easily diagnosis treatment in appropriate time and approach.

\section{Acknowledgment}

The work is supported by the Department of Biomedical Engineering, Faculty of Advanced Medical Sciences, Tabriz University of Medical Sciences. The authors wish to thank Dr. F. Esmaeili, Dr. Z. Reyhani, and Dr. S. Payahoo for providing the dental image database.

\section{Financial support and sponsorship}

This study is funded by Faculty of Advanced Medical Sciences, Tabriz University of Medical Sciences.

\section{Conflicts of interest}

There are no conflicts of interest.

\section{References}

1. White SC, Pharoah MJ. Oral Radiology: Principles and Interpretation. $7^{\text {th }}$ ed. Canada: Elsevier Mosby; 2014. 
2. Cucchiara R, Lamma E, Sansoni T. An image analysis approach for automatically re-orienteering CT images for dental implants. Comput Med Imaging Graph 2004;28:185-201.

3. Hosntalab M, Zoroofi RA, Tehrani-Fard AA, Shirani G. Segmentation of teeth in CT volumetric dataset by panoramic projection and variational level set. Int J Comput Assist Radiol Surg 2008;3:257-65.

4. Momeni M, Zoroofi RA. Automated dental recognition by wavelet descriptors in CT multi-slices data. Int J Comput Assist Radiol Surg 2008;3:533-42.

5. Akhoondali H, Zoroofi R, Shirani G. Rapid automatic segmentation and visualization of teeth in CT-scan data. J Applied Sci 2009;9:2031-44.

6. Sepehrian M, Deylami AM, Zoroofi RA. Individual Teeth Segmentation in CBCT and MSCT Dental Images using
Watershed. Biomedical Engineering (ICBME), 20 ${ }^{\text {th }}$ Iranian Conference on 2013: IEEE; 2013.

7. Mortaheb P, Rezaeian M. Metal artifact reduction and segmentation of dental computerized tomography images using least square support vector machine and mean shift algorithm. J Med Signals Sens 2016;6:1-1.

8. Gonzalez RC, Woods RE. Digital Image Processing. New Jersey: Tom Robbins; 2002.

9. Available from: http://www.mathworks.com.

10. Soille P. Morphological Image Analysis: Principles and Applications. $2^{\text {th }}$ ed. Germany: Springer Science \& Business Media; 2013.

11. Sokolova M, Lapalme G. A systematic analysis of performance measures for classification tasks. Inf Process Manage 2009;45:427-37.

\section{BIOGRAPHIES}

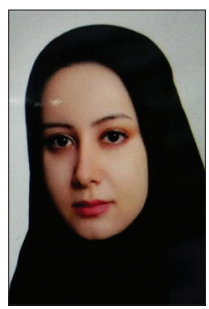

Somayeh Kakehbaraei Received B.A. degree in Pure Mathematic from the University of Kurdestan, Kurdestan, Iran, in 2009, and the M.Sc. degree in Biomedical Engineering (Bioelectric) from Tabriz University of Medical Sciences, Tabriz, Iran in 2018. Her research interests are Biomedical image processing and Computer Vision.

Email: somikakehbaraei@gmail.com

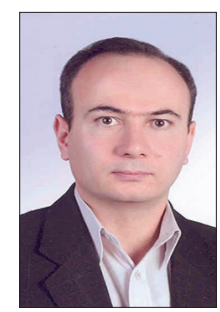

Hadi Seyedarabi Seyedarabi Received B.S. degree from University of Tabriz, Iran, in 1993, the M.S. degree from K.N.T. University of technology, Tehran, Iran in 1996 and Ph.D. degree from University of Tabriz, Iran, in 2006 all in Electrical Engineering. He is currently an associate professor of Faculty of Electrical and
His research interests are Image Processing, Computer Vision, Video Coding, Human-Computer Interaction, Facial Expression Recognition and Facial Animation.

Email: seyedarabi@tabrizu.ac.ir

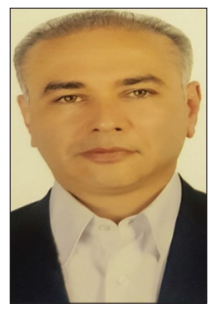

Ali Taghavi Zenouz Received D.D.S. degree from Shahid Beheshti University of Medical Sciences, Tehran, Iran, in 1990, and Oral Med Specialist degree from Tehran University of Medical Sciences, Tehran, Iran, in 1996. He is currently an associate professor of Tabriz University of Medical Sciences, Tabriz, Iran. His research interests are Oral Lichen Planus and Oral Mucosal Lesions.

Email: taghaviz_a@hotmail.com Computer Engineering in University of Tabriz, Tabriz, Iran. 